Discrete Comput Geom 27:419-439 (2002)

DOI: $10.1007 / \mathrm{s} 00454-001-0072-5$

\title{
On a Conjecture of A. Bezdek*
}

\author{
R. N. Karasev \\ Department of Mathematics, Moscow Institute of Physics and Technology, \\ Dolgoprudny, Moscow region, Russia 141700 \\ r_n_karasev@mail.ru
}

\begin{abstract}
The aim of this paper is to prove a conjecture of A. Bezdek. It generalizes conjectures of V. V. Proizvolov. The theorems of this paper are as follows: Suppose points $\left\{x_{i}\right\}_{i=1}^{p}$ and a polytope $X$ are given. Then we can choose $p$ facets $\left\{F_{i}\right\}_{i=1}^{p}$ of $X$ so that the convex hulls conv $\left(\left\{x_{i}\right\} \cup F_{i}\right)$ either cover the polytope or do not intersect pairwise in interior points.
\end{abstract}

\section{Introduction}

Before formulating the main results of the paper we give a brief introduction into the problem. The history of the problem looks as follows:

(1) Proizvolov in [7] raised a "covering" question: Is it true that any $n$ points $A_{1}, \ldots$, $A_{n}$ inside a convex $n$-gon (with sides $s_{1}, \ldots, s_{n}$ ) can be renumbered so that triangles having bases $s_{i}$ and a vertex at $A_{i}$ cover the entire polygon?

(2) Bogomol'naya et al. in [2] had three results:

(a) They proved Proizvolov's conjecture.

(b) They proved the analogous statement for "packing", i.e., they proved that renumbering can be done so that the $n$ triangles are nonoverlapping.

(c) They outlined a proof for the higher-dimensional case for both the packing and covering problems. In this case the $n$-gon is replaced with a polytope with $n$ facets $F_{1}, \ldots, F_{n}, n$ points $A_{1}, \ldots, A_{n}$ are given, and we construct cones with vertex $A_{i}$ and base $F_{i}$ instead of triangles.

(3) Bezdek studied in [1] the generalization of (2) in the plane. In his version the points do not have to be inside the convex polygon:

(a) He proved that with appropriate matching, the triangles still can cover the polygon.

* This research was supported by the Russian Fund of Fundamental Researches Grant No. 00-01-00705. 
(b) He gave a short proof that at least $n / 3$ nonoverlapping triangles can be constructed.

(c) He gave an example where at most $n / 2$ triangles can be constructed and conjectured that $n / 2$ triangles always can be constructed.

The author in this paper improved on both (2c) and (3). Below we give an informal outline of the results. Assume the polytope has $m$ facets and assume $m$ points are given.

About (2c):

(1) With the restriction that the points are inside the polygon the author proved that $m$ nonoverlapping cones can be constructed (Theorem 3 ).

(2) In other words, "points lie inside the polytope" is sufficient to guarantee the existence of $m$ nonoverlapping cones. The author found another sufficient condition for the existence of $m$ nonoverlapping cones in higher dimension (Theorem 4). The condition is a bit technical, it requires that for each subset of the facets, the intersection of the outer halfspaces determined by supporting hyperplanes of these facets contain at least as many points as the number of these facets.

About (3):

(1) Without the restriction that the points are inside the author proved that $m$ cones can be constructed to cover the polytope (Theorem 2 ).

(2) He also proved that $m / 2$ nonoverlapping cones can be constructed in the same case (Theorem 1).

The author also poses a few conjectures at the end of the paper. Informally, they deal with questions such as the following: Is it true that if $\mathbb{R}^{n}$ is partitioned into $m$ convex sets $A_{1}, \ldots, A_{m}$ and $m$ vectors $v_{1}, \ldots, v_{n}$ are given, then one can renumber vectors so that the sets $A_{i}+v_{i}$ do not intersect (as a variant: cover the entire $\mathbb{R}^{n}$ )?

Now we formulate the main results. First, we have to introduce some notation. Suppose $V \subseteq \mathbb{R}^{d}$; then

(1) conv $V$, lin $V$, aff $V$ denote its convex, linear, and affine hull of $V$;

(2) int $V, \operatorname{cl} V$, bd $V,|V|$ denote its interior (relative interior for convex $V$ ), closure, boundary, number of elements of $V$;

(3) $\operatorname{dim}_{l} V, \operatorname{dim}_{a} V$, and codim $V$ denote its linear, affine dimension, and codimension of $V$;

(4) $I_{m}=\{1,2, \ldots, m\}$ denotes the set of indices and $S^{m}$ denotes the group of permutations of $I_{m}$.

Let $L_{1}^{n}$ be a space of linear functions on $\mathbb{R}^{n}$. Suppose $f \in L_{1}^{n}$; by definition, put

(1) $H(f)=\left\{x \in \mathbb{R}^{n}: f(x)=0\right\}$ - the hyperplane;

(2) $H^{-}(f)=\left\{x \in \mathbb{R}^{n}: f(x)<0\right\}$ - the open halfspace;

(3) $H^{+}(f)=\left\{x \in \mathbb{R}^{n}: f(x) \geq 0\right\}$ - the closed halfspace.

Recall that a polyhedral set is an intersection of a family of halfspaces:

$$
X=\bigcap_{i \in I_{m}} H^{+}\left(f_{i}\right) .
$$


Suppose $X$ is a polyhedral set; we may assume that the system $\left\{f_{i}\right\}_{i \in I_{m}}$ is such that

$$
\operatorname{codim} F_{i}=1, \quad F_{i}=H\left(f_{i}\right) \cap X
$$

for any $i \in I_{m}$, and in what follows we assume that this condition holds.

In this case let the facets of $X$ be the sets $F_{i}\left(i \in I_{m}\right)$. Also, put $H_{i}=H^{-}\left(f_{i}\right), i \in I_{m}$. If a polyhedral set is bounded, then it is called a polytope.

Theorem 1. Let $X$ be a polyhedral set in $\mathbb{R}^{n}$, let $\left\{F_{i}\right\}_{i \in I_{m}}$ be its facets, and let $\left\{x_{i}\right\}_{i \in I_{p}} \subset$ $\mathbb{R}^{n}$, where $p=\lceil m / 2\rceil$. Then there exist an injection $\sigma: I_{p} \rightarrow I_{m}$ such that

$$
\text { int } \operatorname{conv}\left(\left\{x_{k}\right\} \cup F_{\sigma(k)}\right) \cap \operatorname{int} \operatorname{conv}\left(\left\{x_{l}\right\} \cup F_{\sigma(l)}\right)=\emptyset \text {. }
$$

The number $p$ cannot be greater. Bezdek in [1] constructed a counterexample for $p>\lceil m / 2\rceil$ and $n=2$. We claim that Theorem 1 is not true for $p>\lceil m / 2\rceil$ and for any $n$. Indeed, further we show that if the statement of Theorem 1 is true for some $n, m$, and $p$, then it is true for any $n^{\prime}<n$ and the same $m$ and $p$. In particular, if $n^{\prime}=2$ we come to a contradiction with the counterexample.

Theorem 2. Let $X$ be a polytope in $\mathbb{R}^{n}$, let $\left\{F_{i}\right\}_{i \in I_{m}}$ be its facets, and let $\left\{x_{i}\right\}_{i \in I_{m}} \subset \mathbb{R}^{n}$. Then there exists $\sigma \in S_{m}$ such that

$$
X \subseteq \bigcup_{i \in I_{m}} \operatorname{conv}\left(\left\{x_{i}\right\} \cup F_{\sigma(i)}\right) .
$$

Theorem 3. Let $X$ be a polyhedral set in $\mathbb{R}^{n}$, let $\left\{F_{i}\right\}_{i \in I_{m}}$ be its facets, and let $\left\{x_{i}\right\}_{i \in I_{m}} \subset$ $X$. Then there exists $\sigma \in S_{m}$ such that

$$
\text { int } \operatorname{conv}\left(\left\{x_{k}\right\} \cup F_{\sigma(k)}\right) \cap \operatorname{int} \operatorname{conv}\left(\left\{x_{l}\right\} \cup F_{\sigma(l)}\right)=\emptyset, \quad l \neq k \in I_{m} .
$$

Theorem 4. Let $X$ be a polyhedral set in $\mathbb{R}^{n}$, let $\left\{F_{i}\right\}_{i \in I_{m}}$ be its facets, and let $\left\{x_{i}\right\}_{i \in I_{m}} \subset$ $\mathbb{R}^{n} \backslash X$. Suppose for any $I \subseteq I_{m}$ we have

$$
\left|\left\{x_{i}\right\}_{i \in I_{m}} \cap \bigcup_{i \in I} H_{i}\right| \geq|I| .
$$

Then there exists $\sigma \in S_{m}$ such that

$$
\text { int } \operatorname{conv}\left(\left\{x_{k}\right\} \cup F_{\sigma(k)}\right) \cap \operatorname{int} \operatorname{conv}\left(\left\{x_{l}\right\} \cup F_{\sigma(l)}\right)=\emptyset, \quad l \neq k \in I_{m},
$$

and

$$
\text { int } \operatorname{conv}\left(\left\{x_{i}\right\} \cup F_{\sigma(i)}\right) \cap \operatorname{int} X=\emptyset, \quad \text { for any } \quad i \in I_{m} \text {. }
$$

The proofs of all the above theorems use the same method, which is different from the methods of [1] and [2]. 


\section{Auxiliary Assertions}

We give some definitions.

Definition 1. We say that the set $V$ in a linear (affine) space $L(A)$ is in general position and write $V \in L G P(V \in A G P)$ if

$$
\operatorname{dim}_{l} U=\min \left\{|U|, \operatorname{dim}_{l} L\right\} \quad\left(\operatorname{dim}_{a} U=\min \left\{|U|-1, \operatorname{dim}_{a} A\right\}\right)
$$

for any finite $U \subseteq V$.

This definition can be generalized.

Definition 2. Suppose $\mathcal{F}$ is a family of maps from some finite set $I$ to $L(A)$, then we say that the map $\lambda \in \mathcal{F}$ is in general position and write $\lambda \in \operatorname{LGP}(\mathcal{F})(\lambda \in A G P(\mathcal{F}))$ if

$$
\operatorname{dim} \lambda(U)=\max _{\lambda^{\prime} \in \mathcal{F}} \operatorname{dim} \lambda^{\prime}(U)
$$

for all $U \subseteq I$.

If the family $\mathcal{F}$ is an irreducible algebraic variety, considered as a subset of $L^{I}\left(A^{I}\right)$, then $L G P(\mathcal{F})(A G P(\mathcal{F}))$ is open and everywhere dense in $\mathcal{F}$.

Definition 3. A polyhedral set $S \subset \mathbb{R}^{n}$ is called simple if either it is a simplex or $S=\bigcap_{i \in I_{m}} H^{+}\left(f_{i}\right)$, where $\left\{f_{i}\right\}_{i \in I_{m}} \in L G P$ and $m \leq n$.

Lemma 1. Let $X$ be a polyhedral set in $\mathbb{R}^{n}$, and let $\left\{F_{i}\right\}_{i \in I_{m}}$ be its facets. Then $X=$ $S \cap L$, where $S$ is a simple polyhedral set in $\mathbb{R}^{N}, L$ is an n-dimensional affine subspace in $\mathbb{R}^{N}$, and $F_{i}=G_{i} \cap L$, where $\left\{G_{i}\right\}_{i \in I_{m}}$ are the facets of $S$. Also, if $X$ is a polytope, then $S$ is a simplex.

This is well known, see for example [3].

Lemma 2. Let $G$ be a finite graph on vertices $V \subseteq L_{1}^{n}$ in general position, let $E=$ $\left\{e_{i}\right\}_{i \in I_{l}}, l \geq|V|$, be its edges, and let $\mathcal{F}$ be the family of all maps $g: E \rightarrow L_{1}^{n}$ such that if $e \in E$ is the edge with endpoints $f^{\prime}$ and $f^{\prime \prime}$, then $H\left(f^{\prime}\right) \cap H\left(f^{\prime \prime}\right) \subseteq H(g(e))$. Then there exists a subset $W \subseteq V(W \neq \emptyset)$ such that for any map $g \in \operatorname{LGP}(\mathcal{F})$,

$$
\bigcap_{i=1}^{l} H\left(g\left(e_{i}\right)\right) \subseteq \bigcap_{f \in W} H(f) .
$$

Proof. Since $|E| \geq|V|, G$ has a cycle. Without loss of generality we may assume that $e_{1}, \ldots, e_{m}$ are the edges of the cycle and $f_{1}, f_{2}, \ldots, f_{m}$ are its vertices. In this case we have

$$
\begin{gathered}
g\left(e_{1}\right)=a_{11} f_{1}+a_{21} f_{2}, \quad g\left(e_{2}\right)=a_{22} f_{2}+a_{32} f_{3}, \ldots, \\
g\left(e_{m-1}\right)=a_{m-1 m-1} f_{m-1}+a_{m m-1} f_{m}, g\left(e_{m}\right)=a_{m m} f_{m}+a_{1 m} f_{1} .
\end{gathered}
$$


Hence $\operatorname{lin}\left\{g\left(e_{k}\right)\right\}_{k=1}^{m} \subseteq \operatorname{lin}\left\{f_{k}\right\}_{k=1}^{m}$ and if $g \in L G P(\mathcal{F})$, then

$$
\operatorname{lin}\left\{g\left(e_{k}\right)\right\}_{k=1}^{m}=\operatorname{lin}\left\{f_{k}\right\}_{k=1}^{m} .
$$

We obtain

$$
\bigcap_{i=1}^{l} H\left(g\left(e_{i}\right)\right) \subseteq \bigcap_{i=1}^{m} H\left(g\left(e_{i}\right)\right)=\bigcap_{i=1}^{m} H\left(f_{i}\right)
$$

Put $W=\left\{f_{1}, f_{2}, \ldots, f_{m}\right\}$. The lemma is proved.

Let $a \neq b \in \mathbb{R}^{n}$, denote by $[a, b],(a, b),\langle a, b\rangle$, and $[a, b\rangle$ the segment between $a$ and $b$, its relative interior, the straight line passing through $a$ and $b$, and the ray, emanating from $a$, passing through $b$. Also, put

$$
\langle a, b\rangle=[a, b\rangle \backslash\{a\} \backslash(a, b) .
$$

This is the ray in $\langle a, b\rangle$, emanating from $b$ and not containing $a$.

Definition 4. A set $U \supseteq V$ is called $V$-starshaped if for any $u \in U$ and $v \in V$ we have $[u, v] \subseteq U$. Clearly, if

(1) $V \neq \emptyset$, then a $V$-starshaped set $U$ is starshaped;

(2) sets $U_{i}, i \in I$, are $V$-starshaped, then $\bigcup_{i \in I} U_{i}$ and $\bigcap_{i \in I} U_{i}$ are $V$-starshaped;

(3) $U$ is $V$-starshaped and $W \subseteq V$, then $U$ is $W$-starshaped.

The proofs of the following lemmas are similar to the topological proof of Helly's theorem (see [4]).

Lemma 3. Let $\left\{V_{i}\right\}_{i=1}^{d}$ be a family of closed subsets of a simplex $S$ in $\mathbb{R}^{d-1}$ with facets $\left\{F_{i}\right\}_{i=1}^{d}$ such that:

(1) $V_{i}$ is $F_{i}$-starshaped;

(2) $S \subseteq \cup_{i=1}^{d} V_{i}$.

Then $\bigcap_{i=1}^{d} V_{i} \neq \varnothing$.

We prove the following generalization.

Lemma 4. Let $S \subseteq \mathbb{R}^{d-1}$ be a homotopically trivial set and let $\left\{F_{i}\right\}_{i=1}^{d}$ be a family of subsets of $S$ such that $\bigcap_{i \in J} F_{i} \neq \emptyset$ is homotopically trivial for any proper subset $J \subset I_{d}$. Let $\left\{V_{i}\right\}_{i=1}^{d}$ be a family of subsets of $S$ such that:

(1) $V_{i}$ is $F_{i}$-starshaped;

(2) $S \subseteq \bigcup_{i=1}^{d} V_{i}$.

Then $\bigcap_{i=1}^{d} V_{i} \neq \emptyset$.

Proof. Suppose $J \subset I_{d}$, denote $V_{J}=\bigcap_{i \in J} V_{i}$ and $F_{J}=\bigcap_{i \in J} F_{i}$. Since the sets $V_{i}$ are $F_{J}$-starshaped, $V_{J}$ is $F_{J}$-starshaped. Hence the set $V_{J}$ is homotopically trivial, and its cohomology $H^{m}\left(V_{J}\right)=0$ for any $m>0$. 
Suppose $\bigcap_{i=1}^{d} V_{i}=\emptyset$. From Leray's theorem (see [5]) for the covering $\mathcal{V}=\left\{V_{i}\right\}$ of the space $S$ we have $H^{i}(S, \mathbb{Z})=H^{i}(\mathcal{V}, \mathbb{Z})$. In particular,

$$
\mathbb{Z}=H^{d-2}(\mathcal{V}, \mathbb{Z})=H^{d-2}(S, \mathbb{Z})=0 .
$$

This contradiction concludes the proof.

Lemma 5. Let $\left\{U_{i}\right\}_{i=1}^{d}$ be a family of open subsets of a simplex $S$ in $\mathbb{R}^{d-1}$ with facets $\left\{F_{i}\right\}_{i=1}^{d}$ such that:

(1) $U_{i} \bigcup$ int $F_{i}$ is int $F_{i}$-starshaped;

(2) int $S \subseteq \bigcup_{i=1}^{d} U_{i}$;

(3) any point $p \in \operatorname{bd} S$ has a neighborhood $N(p)$ such that $N(p) \cap$ int $S \subseteq \bigcup_{i, p \in F_{i}} U_{i}$.

Then $\bigcap_{i=1}^{d} U_{i} \neq \emptyset$.

Proof. We may assume that $S$ is a regular simplex.

Let $A_{i}^{k}$ be a dilation with respect to aff $F_{i}$ with factor $(1-1 / k), k \in \mathbb{N}$. Let

$$
U_{i}^{k}=A_{i}^{k}\left(U_{i}\right), \quad V_{i}^{k}=\operatorname{cl} U_{i}^{k} .
$$

Obviously, $U_{i}^{k} \subseteq U_{i}^{k^{\prime}}$ and $V_{i}^{k} \subseteq V_{i}^{k^{\prime}}$ if $k \leq k^{\prime}$. Since $F_{i} \subseteq \operatorname{cl} U_{i}, F_{i} \subseteq V_{i}^{k}$ for all $i \in I_{d}$ and $k \in \mathbb{N}$.

Consider the families $\mathcal{V}_{k}=\left\{V_{i}^{k}\right\}_{i=1}^{d}, k \in \mathbb{N}$. Since $F_{i} \subseteq \operatorname{cl} U_{i}$ we get $F_{i} \subseteq V_{i}^{k}$. Also, it is clear that $V_{i}^{k} \bigcap$ int $S \subseteq U_{i}$ and $\bigcup_{k} V_{i}^{k} \bigcap$ int $S=U_{i}$.

Let $G=\bigcap_{i \in J_{G}} F_{i}$ be a face of $S$. We prove by induction on $\operatorname{codim} G=d-1-\operatorname{dim}_{a} G$ the following fact: there exists a neighborhood $W_{G}$ of int $G$ such that for $k$ large enough,

$$
W_{G} \cap \operatorname{int} S \subseteq \bigcup_{i \in J_{G}} U_{i}^{k} .
$$

If $\operatorname{codim} G=1$, then $G=F_{i}$ for some $i$. In this case the set

$$
U_{i}^{k} \cup \operatorname{int} F_{i}
$$

is open in topology of the space $S$ and contains int $F_{i}$.

Suppose $\operatorname{codim} G>1$. Take a point $p \in \operatorname{int} G$. By the statement of the lemma there exists a neighborhood $N(p)$ such that

$$
N(p) \cap \operatorname{int} S \subseteq \bigcup_{p \in F_{i}} U_{i}
$$

We may assume that for any $i \notin J_{G}$ we have $N(p) \bigcap F_{i}=\emptyset$.

Let $L_{p}^{\perp}$ be an affine subspace such that $p \in L_{p}^{\perp}, G \perp L_{p}^{\perp}$, and

$$
\operatorname{dim}_{a} L_{p}^{\perp}=d-1-\operatorname{dim}_{a} G .
$$

Take a sphere $\Sigma$ in $L_{p}^{\perp}$ with center in $p$ such that $\Sigma \subset N(p)$. Obviously, if for a face $G^{\prime}$ we have $\Sigma \cap G^{\prime} \neq \emptyset$, then $G \subset G^{\prime}$ and $\operatorname{dim}_{a} G^{\prime}>\operatorname{dim}_{a} G$. By the inductive assumption there exists a neighborhood $W_{G^{\prime}}$ of int $G^{\prime}$ such that for large enough $k$,

$$
W_{G^{\prime}} \cap \operatorname{int} S \subseteq \bigcup_{i \in J_{G^{\prime}}} U_{i}^{k} .
$$


Hence $W=\bigcup_{G \subset G^{\prime}} W_{G^{\prime}}$ is a neighborhood of $\left(\bigcup_{G^{\prime} \supset G} G^{\prime}\right) \cap \Sigma$ and

$$
W \cap \operatorname{int} S \subseteq \bigcup_{G^{\prime} \supseteq G} \bigcup_{i \in J_{G^{\prime}}} U_{i}^{k}
$$

for large enough $k$. The set

$$
Y=(\Sigma \cap \operatorname{int} S) \backslash W=(\Sigma \backslash W) \cap \operatorname{int} S
$$

is closed and, therefore, compact. Since $Y$ is compact and

$$
Y \subseteq N(p) \subseteq \bigcup_{k=1}^{\infty} \bigcup_{i \in J_{G}} U_{i}^{k},
$$

we conclude that $Y \subseteq \bigcup_{i \in J_{G}} U_{i}^{k}$ for large enough $k$. Hence,

$$
\Sigma \cap \operatorname{int} S \subseteq \bigcup_{i \in J_{G}} U_{i}^{k}
$$

for large enough $k$.

Since $U_{i}^{k} \cup$ int $G$ are int $G$-starshaped for $i \in J_{G}$, we obtain

$$
\text { int conv(int } G \cup \Sigma) \cap \operatorname{int} S \subseteq \bigcup_{i \in J_{G}} U_{i}^{k} .
$$

The set int conv(int $G \cup \Sigma$ ) is a neighborhood of int $G$, so the step of induction is made.

Thus $\bigcup_{i \in I_{d}} U_{i}^{k}$ for large enough $k$ contains the intersection of some neighborhood $W \supset$ bd $S$ with int $S$. Since the set int $S \backslash W$ is compact, it is contained in $\bigcup_{i} U_{i}^{k}$ for large enough $k$. Hence int $S \subseteq \bigcup_{i=1}^{d} U_{i}^{k}$ for large enough $k$.

By Lemma 3 for such $k$ we have $\bigcap_{i=1}^{d} V_{i}^{k}=Y \neq \emptyset$. As was shown above, $V_{i}^{k} \cap$ bd $S=$ $F_{i}$, hence $Y \cap$ bd $S=\emptyset$. Then $Y \subseteq \bigcap_{i=1}^{d} U_{i}$. This completes the proof.

\section{Proofs of the Theorems}

Note that it is sufficient to prove Theorems 1-4 for the case of a simple polyhedral set (polytope) $X$.

By Lemma 1, $X=S \cap L$, where $S$ is a simple polyhedral set, $L$ is an $n$-dimensional affine subspace in $\mathbb{R}^{N}$, and $F_{i}=F_{i}^{S} \cap L$, where $\left\{F_{i}^{S}\right\}_{i=i n I_{m}}$ are facets of $S$.

Assume that Theorems 1-4 for $S$ are proved. Then from

$$
\operatorname{conv}\left(\left\{x_{i}\right\} \cup F_{\sigma(i)}\right)=L \cap \operatorname{conv}\left(\left\{x_{i}\right\} \cup F_{\sigma(i)}^{S}\right)
$$

we readily conclude the following: if $S \subseteq \bigcup \operatorname{conv}\left(\left\{x_{i}\right\} \cup F_{\sigma(i)}^{S}\right)$, then $X \subseteq \bigcup \operatorname{conv}\left(\left\{x_{i}\right\} \cup\right.$ $\left.F_{\sigma(i)}\right)$; and if

$$
\operatorname{int} \operatorname{conv}\left(\left\{x_{l}\right\} \cup F_{\sigma(l)}^{S}\right) \cap \operatorname{int} \operatorname{conv}\left(\left\{x_{k}\right\} \cup F_{\sigma(k)}^{S}\right)=\emptyset,
$$


then

$$
\text { int } \operatorname{conv}\left(\left\{x_{l}\right\} \cup F_{\sigma(l)}\right) \cap \operatorname{int} \operatorname{conv}\left(\left\{x_{k}\right\} \cup F_{\sigma(k)}\right)=\emptyset .
$$

Thus the theorems for $X$ are also true.

We consider further a simple polyhedral set $S$; let its facets be $\left\{F_{i}\right\}_{i \in I_{m}}$. The variety of all sets $\left\{x_{i}\right\}_{i \in I_{m}}$, such that $\left\{x_{i}\right\}_{i \in I_{m}} \in A G P$ and $\left\{x_{i}\right\}_{i \in I_{m}}$ are in general position with respect to $\left\{\right.$ aff $\left.F_{i}\right\}$ ( $x_{j} \notin$ aff $F_{i}$ for all $i$ and $j$ ), is open and everywhere dense in the variety of all sets $\left\{x_{i}\right\}_{i \in I_{m}}$. Put by definition

$$
\mathcal{I}=\left\{(i, j, k) \in I_{m} \times I_{m} \times I_{m}: j>k\right\} .
$$

Let a map $g: \mathcal{I} \rightarrow L_{1}^{N}$ be called corresponding to a set $\left\{x_{i}\right\}_{i \in I_{m}} \subseteq \mathbb{R}^{N}$ if

$$
H(g(i, j, k))=H\left(g_{i j k}\right) \supseteq \operatorname{aff}\left(x_{i}, F_{j} \cap F_{k}\right) .
$$

Let $\mathcal{F}$ be the family of all maps $\gamma: I^{3} \rightarrow L_{1}^{n}$ such that

$$
H\left(\gamma_{i j k}\right) \supseteq F_{j} \cap F_{k}, \quad \bigcap_{j>k} H\left(\gamma_{i j k}\right) \neq \emptyset .
$$

It is clear that $g \in \mathcal{F}$ if $g$ corresponds to some $\left\{x_{i}\right\}_{i \in I_{m}}$. Also, any $\gamma \in \mathcal{F}$ corresponds to some set $\left\{x_{i}\right\}_{i \in I_{m}} \subseteq R^{N}$, which can be obtained by taking for all $i \in I_{m}$ some

$$
x_{i} \in \bigcap_{j>k} H\left(\gamma_{i j k}\right)
$$

If $\gamma \in L G P(\mathcal{F})$, then the set $\left\{x_{i}\right\}$ is uniquely determined by $\gamma$. In fact, in this case for any $i \in I_{m}$ the set $\bigcap_{j>k} H\left(\gamma_{i j k}\right)$ contains only one point, since its dimension is minimal over all $\gamma^{\prime} \in \mathcal{F}$.

It follows that the variety of sets $\left\{x_{i}\right\}_{i \in I_{m}}$, such that they have a corresponding $g \in$ $L G P(\mathcal{F})$, is open and everywhere dense in the variety of all sets $\left\{x_{i}\right\}_{i \in I_{m}}$, since the variety of all sets is $R^{N m}$ and is an irreducible algebraic variety.

We claim that it is sufficient to prove Theorems $2-4$ for an everywhere dense subvariety of the variety of all sets $\left\{x_{i}\right\}_{i \in I_{m}}$. Let

$$
\lim _{k}\left\{x_{i}^{(k)}\right\}_{i \in I_{m}}=\left\{x_{i}\right\}_{i \in I_{m}},
$$

and for the set $\left\{x_{i}^{(k)}\right\}_{i \in I_{m}}$ the statement of some of the theorems is true. For an infinite number of $k \in \mathbb{N}$ the permutation (injection) $\sigma$ (and the set $J$ from Theorem 1) is the same. We may assume that it is the same for all $k \in \mathbb{N}$. Then

$$
\operatorname{conv}\left(\left\{x_{i}\right\} \cup F_{\sigma(i)}\right)=\lim _{k} \operatorname{conv}\left(\left\{x_{i}^{(k)}\right\} \cup F_{\sigma(i)}\right) .
$$

In the case of Theorem 2 the sets $\operatorname{conv}\left(\left\{x_{i}\right\} \cup F_{\sigma(i)}\right)$ are closed and if

$$
\bigcup_{i \in I_{m}} \operatorname{conv}\left(\left\{x_{i}\right\} \cup F_{\sigma(i)}\right) \not \ngtr x \in S,
$$


then

$$
\operatorname{conv}\left(\left\{x_{i}^{(k)}\right\} \cup F_{\sigma(i)}\right) \not \ngtr x
$$

for large enough $k$.

In the case of other theorems if a pair of sets int $\operatorname{conv}\left(\left\{x_{i}\right\} \cup F_{\sigma(i)}\right)$ has a common point $x$, then for large enough $k$ the corresponding pair int $\operatorname{conv}\left(\left\{x_{i}^{(k)}\right\} \cup F_{\sigma(i)}\right)$ contains it too.

In the case of Theorem 4, if $x \in \operatorname{int} \operatorname{conv}\left(\left\{x_{i}\right\} \cup F_{\sigma(i)}\right) \cap$ int $S$ for some $i$, then

$$
x \in \operatorname{int} \operatorname{conv}\left(\left\{x_{i}^{(k)}\right\} \cup F_{\sigma(i)}\right) \cap \operatorname{int} S .
$$

Thus for $\left\{x_{i}\right\}_{i \in I_{m}}$ the theorems are also true.

We prove Theorems $2-4$ under the following conditions:

(1) $S$ is a simple polyhedral set with facets $\left\{F_{i}\right\}_{i \in I_{m}}$;

(2) the points $\left\{x_{i}\right\}_{i \in I_{m}} \in A G P$ and they are in general position with respect to facets of $S$;

(3) the map $g$ that corresponds to $\left\{x_{i}\right\}_{\in I_{m}}$ is in $L G P(\mathcal{F})$.

This will be sufficient, since the variety of sets $\left\{x_{i}\right\}_{i \in I_{m}}$ that satisfy the conditions is everywhere dense in the variety of all sets. Note that under these conditions

$$
H\left(g_{i j k}\right)=\operatorname{aff}\left(x_{i}, F_{j} \cap F_{k}\right),
$$

since the right side is a subset of the left side and their dimensions coincide.

Next we prove Theorem 2, then Theorems 3 and 4, and finally using the last two theorems we prove Theorem 1.

Proof of Theorem 2. Here $S$ is a simplex, since $X$ is a polytope. We use induction on $\operatorname{dim} S$. For $\operatorname{dim}_{a} S=1$ the theorem is obvious. Consider the following condition:

For any $I \subseteq I_{m}$ such that $I \neq \emptyset$,

$$
\left|\left\{x_{j}\right\}_{j \in I_{m}} \cap \bigcap_{i \in I} H_{i}\right|<m-|I| .
$$

We consider two cases.

Case 1: condition (*) fails. Then there exist $I \subseteq I_{m}$ and $J \subseteq I_{m}$ such that $|J| \geq m-|I|$ and $f_{i}\left(x_{j}\right)<0$ for all $i \in I$ and $j \in J$. Denote $A=\bigcap_{i \in I} F_{i}, B=\bigcap_{i \notin I} F_{i}$.

Let $L=\operatorname{aff} A$. We show that $\operatorname{conv}\left(\left\{x_{j}\right\} \cup B\right) \cap L \neq \emptyset$ for any $j \in J$. Let $p$ be a projection along $L$. Then $p(L)$ is a point and $p(S)$ is a simplex with a vertex $p(A)$ and a facet $p(B)$. We see that

$$
\left[p\left(x_{j}\right), p(A)\right\rangle \cap p(S) \neq\{p(A)\} .
$$

Indeed, $f_{i}\left(x_{j}\right)<0, f_{i}(A)=0(i \in I, j \in J)$, and the system of inequalities

$$
f_{i}\left(p^{-1}(y)\right) \geq 0, \quad i \in I,
$$


determines $p(S)$ in some neighborhood of $p(A)$. Hence,

$$
\left[p\left(x_{j}\right), p(A)\right\rangle \cap p(B) \neq \emptyset,
$$

therefore,

$$
p(L)=p(A) \in \operatorname{conv}\left(\left\{p\left(x_{j}\right)\right\} \cup p(B)\right)=p\left(\operatorname{conv}\left(\left\{x_{j}\right\} \cup B\right)\right)
$$

and $\operatorname{conv}\left(\left\{x_{j}\right\} \cup B\right) \cap L \neq \emptyset$.

If $|J|>m-|I|$, then take $J^{\prime} \subseteq J$ so that $\left|J^{\prime}\right|=m-|I|$. For any $j \in J^{\prime}$ take any $x_{j}^{\prime} \in L \cap \operatorname{conv}\left(\left\{x_{j}\right\} \cup B\right)$. If $\operatorname{dim}_{a} A=0$, then $J^{\prime}=\{j\}$ and

$$
\operatorname{conv}\left(\left\{x_{j}\right\} \cup B\right) \supseteq \operatorname{conv}(A \cup B)=S .
$$

In this case the theorem is proved.

If $\operatorname{dim}_{a} A>0$, then $A$ and $\left\{x_{j}^{\prime}\right\}_{j \in J^{\prime}}$ satisfy the inductive assumption, since $A$ has $m-|I|=\left|J^{\prime}\right|<m$ facets.

By the inductive assumption we obtain an injection $\sigma: J^{\prime} \rightarrow I_{m}$ such that

$$
\bigcup_{j \in J^{\prime}} \operatorname{conv}\left(\left\{x_{j}^{\prime}\right\} \cup F_{\sigma(j)}^{A}\right) \supseteq A,
$$

where $F_{j}^{A}$ are facets of $A$.

We extend $\sigma$ to $I_{m} \backslash J^{\prime}$ so that it becomes a permutation of $I_{m}$. Then $x_{j}^{\prime} \in L \cap$ $\operatorname{conv}\left(\left\{x_{j}\right\} \cup B\right)$ and

$$
\operatorname{conv}\left(\left\{x_{j}^{\prime}\right\} \cup F_{\sigma(j)}\right) \supseteq \operatorname{conv}\left(\left\{x_{j}\right\} \cup B\right),
$$

therefore,

$$
\operatorname{conv}\left(\left\{x_{j}\right\} \cup F_{\sigma(j)}\right) \supseteq \operatorname{conv}\left(\left\{x_{j}^{\prime}\right\} \cup F_{\sigma(j)}\right) .
$$

Hence for any $j \in J^{\prime}, \operatorname{conv}\left(\left\{x_{j}\right\} \cup F_{\sigma(j)}\right) \supseteq B$ and

$$
\bigcup_{j \in J^{\prime}} \operatorname{conv}\left(\left\{x_{j}\right\} \cup F_{\sigma(j)}\right) \supseteq A .
$$

We obtain

$$
\bigcup_{j \in I_{m}} \operatorname{conv}\left(\left\{x_{j}\right\} \cup F_{\sigma(j)}\right) \supseteq \bigcup_{j \in J^{\prime}} \operatorname{conv}\left(\left\{x_{j}\right\} \cup F_{\sigma(j)}\right) \supseteq \operatorname{conv}(A \cup B)=S .
$$

In this case the proof is complete.

Case 2: condition (*) holds. Denote

$$
U_{i}=\operatorname{int} S \cap \bigcup_{j}\left(\operatorname{conv}\left(\left\{x_{j}\right\} \cup \operatorname{int} F_{i}\right) \backslash\left\{x_{j}\right\}\right) .
$$

A point $x \in \operatorname{int} S$ is in $U_{i}$ iff there exists $y \in \operatorname{int} F_{i}$ and $j \in I_{m}$ such that $x \in\left(x_{j}, y\right)$.

We show that $\left\{U_{i}\right\}_{i \in I_{m}}$ satisfy the conditions of Lemma 5.

Obviously, the sets $U_{i}$ are open. 
Condition (1) holds. Indeed, if $x \in U_{i}, y \in$ int $F_{i}$, and $x^{\prime} \in(x, y)$, then since $x \in U_{i}$ there exists $z \in \operatorname{int} F_{i}$ such that $x \in\left(x_{j}, z\right)$. Then $\left\langle x_{j}, x^{\prime}\right\rangle \cap[z, y]=z^{\prime} \in$ int $F_{i}$ and $x^{\prime} \in\left(x_{j}, z^{\prime}\right)$.

Consider condition (3). Take a point $p \in$ bd $S$. Let $I=\left\{i: p \in F_{i}\right\}$. Condition (3) is equivalent to the fact that for any $x \in \operatorname{int} S \cap N(p)$ there is $j \in I_{m}$ such that \rangle $\left.x_{j}, x\right\rangle \cap$ int $F_{i} \neq \varnothing$ for some $i \in I$.

Let $\left.\left.J=\{j:\rangle x_{j}, p\right\rangle \cap S=\{p\}\right\}$. For some neighborhood $N(p)$ of $p$ we have

$$
S \cap N(p)=\left\{x \in N(p): \forall i \in I f_{i}(x) \geq 0\right\} .
$$

If $j \in I_{m} \backslash J$, then there exists $\left.x \in \backslash x_{j}, p\right\rangle \backslash\{p\}$ such that for any $i \in I, f_{i}(x) \geq 0$. From $f_{i}(p)=0(i \in I)$ it follows that $f_{i}\left(x_{j}\right)<0(i \in I)$, since $f_{i}\left(x_{j}\right) \neq 0$ because of general position. Then

$$
\left\{x_{j}\right\}_{j \in I_{m}} \cap \bigcap_{i \in I} H_{i} \supseteq\left\{x_{j}\right\}_{j \in I_{m} \backslash J}
$$

and condition $(*)$ gives $\left|I_{m} \backslash J\right|<m-|I|$.

Then $|J| \geq|I|+1$. For any $j \in I_{m}$ the point $\left.\left.y_{j}(x)=\right\rangle x_{j}, x\right\rangle \cap$ bd $S$ is a continuous function of $x \in S$. Since $y_{j}(p)=p(j \in J)$, there exists a neighborhood $N(p)$ of $p$ such that for any $x \in N(p), j \in J$, and $i \notin I$ we have $y_{j}(x) \notin F_{i}$.

We show that any $x \in N(p) \cap$ int $S$ is in $U_{i}$ for some $i \in I$. It is sufficient to show that $y_{j}(x) \in \operatorname{int} F_{i}$ for some $j \in J$ and $i \in I$, since in this case $x \in\left(y_{j}, x_{j}\right)$ and $x \in U_{i}$.

Assume the contrary that $y_{j}(x) \notin$ int $F_{i}$ for all $i \in I$ and $j \in J$. Then for any $j \in J$,

$$
y_{j}(x) \in F_{s(j)} \cap F_{t(j)}, \quad s(j), t(j) \in I .
$$

This implies that $x \in \bigcap_{j \in J}$ aff $\left(x_{j}, F_{s(j)} \cap F_{t(j)}\right)$. We obtain that

$$
\operatorname{aff}\left(x_{j}, F_{s(j)} \cap F_{t(j)}\right)=H\left(g_{j s(j) t(j)}\right),
$$

where $g$ is corresponding to $\left\{x_{i}\right\}_{i \in I_{m}}$ and

$$
\left\{\text { aff } F_{s(j)}, \text { aff } F_{t(j)}\right\}_{j \in J} \subseteq\left\{H\left(f_{i}\right)\right\}_{i \in I} .
$$

Denote the family of maps

$$
\mathcal{F}^{\prime}=\left\{\gamma: J \rightarrow L_{1}^{N}: H(\gamma(j)) \supseteq F_{s(j)} \cap F_{t(j)}\right\} .
$$

Since the map of restriction $\mathcal{F} \rightarrow \mathcal{F}^{\prime}(g(i, j, k) \mapsto g(i, s(i), t(i)))$ is surjective, the restriction $g^{\prime}: j \mapsto g_{j s(j) t(j)}$ of $g \in L G P(\mathcal{F})$ is in $L G P\left(\mathcal{F}^{\prime}\right)$.

Thus the set $\left\{f_{i}\right\}_{i \in I}$, its edges $\left(f_{s(j)}, f_{t(j)}\right)(j \in J)$, and the map $g^{\prime}$ satisfy the conditions of Lemma 2 , since $|J| \geq|I|$. In this case $\bigcap_{j \in J} H\left(g_{j}^{\prime}\right) \subseteq H\left(f_{i}\right)=$ aff $F_{i}$ for some $i$, and therefore

$$
x \in \bigcap_{j \in J} H\left(g_{j}^{\prime}\right) \subseteq \text { aff } F_{i}
$$

and $x \in$ int $S$. This is a contradiction.

Condition (2) of Lemma 5 holds too. As above if for some $x \in \operatorname{int} S, y_{j}(x) \notin$ int $F_{i}$ for all $i \in I_{m}$ and $j \in I_{m}$, then $x$ is in the intersection of $m$ hyperplanes $\operatorname{aff}\left(x_{j}, \operatorname{aff} F_{s(j)} \cap\right.$ 
aff $\left.F_{t(j)}\right)$. Applying Lemma 2 to the graph on $m$ vertices with $m$ edges we obtain that $x \in$ aff $F_{i}$ for some $i \in I_{m}$ and $x \in \operatorname{int} S$. This is a contradiction.

By Lemma 5 there exists $x \in \bigcap_{i} U_{i}$. Then for any $i \in I_{m}$ there is $j \in I_{m}$ and $y_{j} \in$ int $F_{i}$ such that $x \in\left(x_{j}, y_{j}\right)$. It means that the rays $\left.\rangle x_{j}, x\right\rangle\left(j \in I_{m}\right)$ intersect interiors of all facets. Since the numbers of the rays and the facets are the same and one ray only intersects one interior of a facet, we get a one-to-one correspondence $\sigma$. Then for any $j \in I_{m}, x \in \operatorname{conv}\left(\left\{x_{j}\right\} \cup\right.$ int $\left.F_{\sigma(j)}\right)$.

Since $S \subseteq \bigcup_{j} \operatorname{conv}\left(\{x\} \cup F_{\sigma(j)}\right)$ and

$$
\operatorname{conv}\left(\left\{x_{j}\right\} \cup F_{\sigma(j)}\right) \supseteq \operatorname{conv}\left(\{x\} \cup F_{\sigma(j)}\right),
$$

we obtain $S \subseteq \bigcup_{j} \operatorname{conv}\left(\left\{x_{j}\right\} \cup F_{\sigma(j)}\right)$.

Proof of Theorem 3. First consider the case when $S$ is a simplex.

Denote

$$
V_{i}=\left\{x \in S: \operatorname{int} \operatorname{conv}\left(\{x\} \cup F_{i}\right) \cap\left\{x_{j}\right\}_{j \in I_{m}}=\emptyset\right\} .
$$

Obviously, the sets $\left\{V_{i}\right\}_{i \in I_{m}}$ are closed. If $x \in V_{i}, y \in F_{i}$, and $x^{\prime} \in[x, y]$, then

$$
\text { int } \operatorname{conv}\left(\left\{x^{\prime}\right\} \cup F_{i}\right) \subseteq \text { int } \operatorname{conv}\left(\{x\} \cup F_{i}\right) .
$$

Thus $V_{i}$ is $F_{i}$-starshaped.

Consider two cases.

Case 1: $S \subseteq \bigcup_{i} V_{i}$. By Lemma 3 we get some $x \in \bigcap_{i} V_{i}$.

Since $x_{j} \notin \operatorname{int} \operatorname{conv}\left(\{x\} \cup F_{i}\right)$ for all $i$ and $j$, we see that for any $j \in I_{m}$,

$$
x_{j} \in \operatorname{conv}\left(\{x\} \cup\left(\operatorname{aff} F_{s(j)} \cap \operatorname{aff} F_{t(j)}\right)\right) .
$$

Let

$$
I=\left\{i: x \notin F_{i}\right\}
$$

We have $s(j), t(j) \in I$ for any $j \in I_{m}$, since otherwise $x_{j} \in F_{i}$, which is false. In other words,

$$
x \in \bigcap_{j \in I_{m}} \operatorname{aff}\left(x_{j}, \text { aff } F_{s(j)} \cap \operatorname{aff} F_{t(j)}\right) .
$$

As in the proof of Theorem 2 we apply Lemma 2 so that $\left\{f_{i}\right\}_{i \in I}$ are the vertices, $\left(f_{s(j)}, f_{t(j)}\right)\left(j \in I_{m}\right)$ are the edges, and the restriction $g^{\prime}(j)=g_{j s(j) t(j)}$ of the map $g$ is the map $g$ in the lemma. Then we obtain $x \in$ aff $F_{i}$ for some $i \in I$. This is a contradiction with the definition of $I$.

Thus case 1 is impossible.

Case 2: $S \nsubseteq \bigcup_{i} V_{i}$. Let $x \in S \backslash \bigcup_{i} V_{i}$. Then for any $i \in I_{m}$ there is $j \in I_{m}$ such that $x_{j} \in \operatorname{int} \operatorname{conv}\left(\{x\} \cup F_{i}\right)$.

The sets int $\operatorname{conv}\left(\{x\} \cup F_{i}\right)$ do not intersect pairwise, hence there is a one-to-one correspondense $\sigma$ such that $x_{j} \in \operatorname{int} \operatorname{conv}\left(\{x\} \cup F_{\sigma(j)}\right)$.

Since

$$
\text { int } \operatorname{conv}\left(\left\{x_{j}\right\} \cup F_{\sigma(j)}\right) \subseteq \operatorname{int} \operatorname{conv}\left(\{x\} \cup F_{\sigma(j)}\right),
$$


we get

$$
\text { int } \operatorname{conv}\left(\left\{x_{k}\right\} \cup F_{\sigma(k)}\right) \cap \operatorname{int} \operatorname{conv}\left(\left\{x_{l}\right\} \cup F_{\sigma(l)}\right)=\emptyset, \quad k \neq l \in I_{m} .
$$

Thus the proof is complete, if $S$ is a simplex.

Suppose $S$ is not a simplex. Then $\bigcap_{i \in I_{m}} F_{i} \neq \emptyset$.

Consider two cases.

Case $1 . \bigcap_{i \in I_{m}} F_{i}=\{v\}$. Take an intersection $S^{\prime}=S \cap h$, where $h$ is a hyperplane, such that $S^{\prime}$ is a simplex with facets

$$
F_{i}^{\prime}=F_{i} \cap h, \quad i \in I_{m} .
$$

Let the central projection from $v$ to $h$ take $x_{i}$ to $x_{i}^{\prime}\left(i \in I_{m}\right)$. The theorem is already proved for $\left\{x_{i}^{\prime}\right\}_{i \in I_{m}}$ and $S^{\prime}$; thus we obtain a permutation $\sigma$.

Let $S_{i}=\operatorname{int} \operatorname{conv}\left(\left\{x_{i}^{\prime}\right\} \cup F_{\sigma(i)}^{\prime}\right)$. Then $S_{l} \cap S_{k}=\emptyset$ for any $k \neq l \in I_{m}$. Take the cone $C_{i}$ formed by the rays from $v$ that intersect $S_{i}$ without the point $v$. Denote $r_{i}=\left[v, x_{i}\right\rangle$ $\left(r_{i} \ni x_{i}^{\prime}\right)$. We see that $C_{i}=\operatorname{int} \operatorname{conv}\left(r_{i} \cup F_{\sigma(i)}\right)$. Obviously, $C_{k} \cap C_{l}=\emptyset$ for all $k \neq l \in I_{m}$. Since

$$
\text { int } \operatorname{conv}\left(\left\{x_{i}\right\} \cup F_{\sigma(i)}\right) \subseteq \operatorname{int} \operatorname{conv}\left(r_{i} \cup F_{\sigma(i)}\right)=C_{i},
$$

we obtain

$$
\text { int } \operatorname{conv}\left(\left\{x_{k}\right\} \cup F_{\sigma(k)}\right) \cap \operatorname{int} \operatorname{conv}\left(\left\{x_{l}\right\} \cup F_{\sigma(l)}\right)=\emptyset, \quad k \neq l \in I_{m} .
$$

Case 2: $\operatorname{dim}_{a} L>0$, where $L=\bigcap_{i} F_{i}$. Let $p$ be a projection along $L$. Then $p(L)$ is a point. The previous case gives an intersection $\sigma$ such that for all $k \neq l \in I_{m}$,

$$
\text { int } \operatorname{conv}\left(\left\{p\left(x_{k}\right)\right\} \cup p\left(F_{\sigma(k)}\right)\right) \cap \operatorname{int} \operatorname{conv}\left(\left\{p\left(x_{l}\right)\right\} \cup p\left(F_{\sigma(l)}\right)\right)=\emptyset .
$$

Then obviously

$$
\text { int } \operatorname{conv}\left(\left\{x_{k}\right\} \cup F_{\sigma(k)}\right) \cap \operatorname{int} \operatorname{conv}\left(\left\{x_{l}\right\} \cup F_{\sigma(l)}\right)=\emptyset, \quad k \neq l \in I_{m} .
$$

Proof of Theorem 4. For any $i \in I_{m}$ denote

$$
U_{i}=\left\{x \in \operatorname{int} S: \exists j \in I_{m}:\left[x, x_{j}\right] \cap \operatorname{int} F_{i} \neq \emptyset\right\} .
$$

The sets $U_{i}$ are open.

We show that $\left\{U_{i}\right\}_{i \in I_{m}}$ satisfy the conditions of Lemma 5.

Condition (1) holds. Let $x \in U_{i}, y \in \operatorname{int} F_{i}$, and $x^{\prime} \in[x, y]$. Let $z=\left[x, x_{j}\right] \cap \operatorname{int} F_{i}$. The points $x^{\prime}$ and $x_{j}$ are on different sides of the hyperplane aff $F_{i}$; then denote $z^{\prime}=$ $\left[x^{\prime}, x_{j}\right] \cap$ aff $F_{i}$. Hence $z^{\prime} \in[z, y]$ and $z^{\prime} \in \operatorname{int} F_{i}$.

Consider condition (3). Let

$$
p \in \operatorname{bd} S, \quad I=\left\{i: p \in F_{i}\right\} .
$$

We prove that there is a neighborhood $N(p)$ of the point $p$ such that

$$
N(p) \cap \operatorname{int} S \subseteq \bigcup_{i \in I} U_{i},
$$


or, equivalently, for any $x \in \operatorname{int} S \cap N(p)$ we have $\left[x_{j}, x\right] \cap \operatorname{int} F_{i} \neq \emptyset$ for some $j \in I_{m}$ and $i \in I$.

Denote $J=\left\{j: x_{j} \in \bigcup_{i \in I} H_{i}\right\}$. Recall that $|J| \geq|I|$ in this theorem. Thus for any $j \in J,\left[x_{j}, p\right] \cap$ bd $S=\{p\}$. For any $j \in I_{m}$ and $x \in S$ denote

$$
y_{j}(x)=\text { the farthest point of }\left[x_{j}, x\right] \cap \operatorname{bd} S \text { from } x .
$$

The points $y_{j}(x)$ are continuous functions of $x \in S$. Since $y_{j}(p)=p(j \in J)$, there is a neighborhood $N(p)$ such that for all $x \in N(p), j \in J$, and $i \notin I$, we have $y_{j}(x) \notin F_{i}$.

We shall show that for any $x \in N(p) \cap$ int $S$ there exists $i \in I$ such that $x \in U_{i}$.

Assume the contrary. For some $x \in N(p) \cap$ int $S$ and any $j \in J$ we have $y_{j}(x) \in$ $F_{s(j)} \cap F_{t(j)}$. In this case the point $x$ is in the intersection of $|J|$ hyperplanes aff $\left(x_{j}\right.$, aff $F_{s(j)} \cap$ aff $\left.F_{t(j)}\right)(j \in J, s(j), t(j) \in I)$. As in the proof of Theorem 2, by Lemma 2 we get a contradiction, since $|J| \geq|I|$.

We show that int $S \subseteq \bigcup_{i \in I_{m}} U_{i}$.

If for some $x$ for all $i \in I_{m}$ and $j \in I_{m}$ we have $y_{j}(x) \notin \operatorname{int} F_{i}$, then $x$ is in the intersection of $m$ hyperplanes $\operatorname{aff}\left(x_{j}, \operatorname{aff} F_{s(j)} \cap \operatorname{aff} F_{t(j)}\right)$. By Lemma 2 this is a contradiction.

If $S$ is a simplex, then by Lemma $5 \bigcap_{i} U_{i} \neq \varnothing$.

If $S$ is such that $\bigcap_{i} F_{i}=\{v\}$, then take an intersection $S^{\prime}=S \cap h$ with a hyperplane $h$ such that $S^{\prime}$ is a simplex. We can apply Lemma 5 to $S^{\prime}$ and the sets $U_{i}^{\prime}=U_{i} \cap h$. As above we see that $\bigcap_{i} U_{i}^{\prime} \neq \emptyset$, and therefore $\bigcap_{i} U_{i} \neq \emptyset$.

In both previous cases we take a point $x \in \bigcap_{i} U_{i}$. The points $\left\{y_{j}(x)\right\}_{j \in I_{m}}$ pierce every int $F_{i}\left(i \in I_{m}\right)$,

$$
\left|\left\{y_{j}(x)\right\}_{j \in I_{m}}\right|=\mid\left\{\text { int } F_{i}\right\}_{i \in I_{m}} \mid,
$$

and int $F_{k} \cap \operatorname{int} F_{l}=\emptyset$ for $k \neq l \in I_{m}$. Thus there is a one-to-one correspondence $\sigma$ such that $y_{j}(x) \in \operatorname{int} F_{\sigma(j)}$ for all $j \in I_{m}$.

Let $C_{j}$ be the cone of rays $[x, y\rangle$, where $y \in \operatorname{int} F_{\sigma(j)}$ without the point $x$. For all $k \neq l \in I_{m}, C_{k} \cap C_{l}=\emptyset$ and $x_{j} \in C_{j}$, since int $F_{\sigma(j)} \ni y_{j}(x) \in\left[x_{j}, x\right]$. Since for all $k \neq l \in I_{m}$, int $\operatorname{conv}\left(\left\{x_{j}\right\} \cup F_{\sigma(j)}\right) \subseteq C_{j}$, we have

$$
\text { int } \operatorname{conv}\left(\left\{x_{k}\right\} \cup F_{\sigma(k)}\right) \cap \operatorname{int} \operatorname{conv}\left(\left\{x_{l}\right\} \cup F_{\sigma(l)}\right)=\emptyset .
$$

Since $x_{j}$ and $S$ are on different sides of aff $F_{\sigma(j)}$ for any $j \in I_{m}$, we have

$$
\text { int } \operatorname{conv}\left(\left\{x_{j}\right\} \cup F_{\sigma(j)}\right) \cap \text { int } S=\emptyset .
$$

The last case is when $\bigcap_{i} F_{i}$ is a linear subspace $L$. As in the previous proof take a projection $p$ along $L$. By the same argument we can deduce the theorem for this case by applying Theorem 3 for the projection.

Proof of Theorem 1. For all $I \subseteq I_{m}$ put

$$
C_{I}=\left\{x: f_{i}(x) \geq 0, \forall i \in I\right\}=\bigcap_{i \in I} H^{+}\left(f_{i}\right) .
$$

Denote $\Phi_{I}^{j}=\left\{i \in I: x_{j} \in H_{i}\right\}$. Equivalently, $\Phi_{I}^{j}=\left\{i \in I: f_{i}\left(x_{j}\right)<0\right\}$. 
Put $I^{0}=I_{m}$. We pass from $I^{s}$ to $I^{s+1}$ as follows. If there exists $Q \subseteq I_{p}$ such that $\left\{x_{j}\right\}_{j \in Q} \cap C_{I^{s}}=\emptyset$ and $\left|\bigcup_{j \in Q} \Phi_{I^{s}}^{j}\right|<|Q|$, then $I^{s+1}=I^{s} \backslash \bigcup_{j \in Q} \Phi_{I^{s}}^{j}$. Then the points $\left\{x_{i}\right\}_{i \in Q}$ get into $C_{I^{s+1}}$.

Since the number of points $x_{j}$ in $C_{I^{s}}$ increases, the sequence $\left\{I^{s}\right\}_{s \geq 0}$ terminates at some $I$. Put by definition

$$
A=\left\{i \in I_{p}: x_{i} \notin C_{I}\right\}, \quad B=I_{p} \backslash A .
$$

Note that the sets $\Phi_{I}^{j}(j \in A)$ satisfy the conditions of Hall's theorem (see [6]) about a system of different representatives.

We have $|I| \geq m-p+1 \geq p$, since when some $n=|Q|$ points $\left\{x_{j}\right\}_{j \in Q}$ got into $C_{I^{s+1}}$, we had $\left|I^{s+1}\right| \geq\left|I^{s}\right|-n+1$, and at last no more than $p$ points got into $C_{I}$. Then by Hall's theorem there exists an injection $r_{0}: A \rightarrow I$ such that $f_{r_{0}(j)}\left(x_{j}\right)<0$ for all $j \in A$. Denote $J=r_{0}(A)$. Denote the facets of $C_{J}$ as $\left\{F_{i}^{J}\right\}_{i \in J}$. The points $\left\{x_{j}\right\}_{j \in A}$ and $C_{J}$ satisfy the conditions of Theorem 4, since for any $L \subseteq J$ we have $\bigcup_{i \in L} H_{i} \supseteq\left\{x_{r_{0}^{-1}(i)}\right\}_{i \in L}$.

Theorem 4 gives a bijection $r: A \rightarrow J$ such that for any $j_{1} \neq j_{2} \in A$,

$$
\text { int } \operatorname{conv}\left(\left\{x_{j_{1}}\right\} \cup F_{r\left(j_{1}\right)}^{J}\right) \cap \operatorname{int} \operatorname{conv}\left(\left\{x_{j_{2}}\right\} \cup F_{r\left(j_{2}\right)}^{J}\right)=\emptyset \text {, }
$$

and for all $j \in A$,

$$
\text { int } \operatorname{conv}\left(\left\{x_{j}\right\} \cup F_{r(j)}^{J}\right) \cap \operatorname{int} C_{J}=\emptyset .
$$

Since $F_{i}^{J} \supseteq F_{i}$, for any $j_{1} \neq j_{2} \in A$ we have

$$
\text { int } \operatorname{conv}\left(\left\{x_{j_{1}}\right\} \cup F_{r\left(j_{1}\right)}\right) \cap \operatorname{int} \operatorname{conv}\left(\left\{x_{j_{2}}\right\} \cup F_{r\left(j_{2}\right)}\right)=\emptyset .
$$

Take $K=I \backslash J$. Let the facets of $C_{K}$ be $\left\{F_{j}^{K}\right\}_{j \in K}$. Consider the set $V=\left\{x_{j}\right\}_{j \in B}$. It clear that $V \subset C_{I} \subseteq C_{K}$ and $|V|=|B|=p-|A|=p-|J| \leq|I|-|J|=|K|$. If $|V|<|K|$, then take $W \supseteq V$, such that $|W|=|K|$, else put $W=V$.

By Theorem 3 for the set of points $W$ and $C_{K}$ we get a bijection $q_{W}: W \rightarrow K$. Let the injection $q: B \rightarrow K$ take any $j \in B$ to $q_{W}\left(x_{j}\right)$. Then for all $j_{1} \neq j_{2} \in B$,

$$
\text { int } \operatorname{conv}\left(\left\{x_{j_{1}}\right\} \cup F_{q\left(j_{1}\right)}^{K}\right) \cap \operatorname{int} \operatorname{conv}\left(\left\{x_{j_{2}}\right\} \cup F_{q\left(j_{2}\right)}^{K}\right)=\emptyset .
$$

Since $F_{i}^{K} \supseteq F_{i}$,

$$
\text { int } \operatorname{conv}\left(\left\{x_{j_{1}}\right\} \cup F_{q\left(j_{1}\right)}\right) \cap \operatorname{int} \operatorname{conv}\left(\left\{x_{j_{2}}\right\} \cup F_{q\left(j_{2}\right)}\right)=\emptyset
$$

for any $j_{1} \neq j_{2} \in B$.

We see that

$$
\text { int } \operatorname{conv}\left(\left\{x_{j_{1}}\right\} \cup F_{r\left(j_{1}\right)}\right) \subseteq \mathbb{R}^{N} \backslash C_{J} \subseteq \mathbb{R}^{N} \backslash C_{I} .
$$

Since $\left\{x_{j}\right\}_{j \in B} \subseteq C_{I}$ and $F_{q(j)} \subseteq C_{I}$ for any $j \in B$, it follows that

$$
\text { int } \operatorname{conv}\left(\left\{x_{j_{2}}\right\} \cup F_{q\left(j_{2}\right)}\right) \subseteq C_{I} .
$$

Hence for any $j_{1} \in A, j_{2} \in B$,

$$
\text { int } \operatorname{conv}\left(\left\{x_{j_{1}}\right\} \cup F_{r\left(j_{1}\right)}\right) \cap \operatorname{int} \operatorname{conv}\left(\left\{x_{j_{2}}\right\} \cup F_{q\left(j_{2}\right)}\right)=\emptyset \text {. }
$$

Let $\sigma$ be defined by $\left.\sigma\right|_{A}=r$ and $\left.\sigma\right|_{B}=q$. This construction of $\sigma$ completes the proof. 


\section{Conclusion}

We deduce the lemma of [2] (stated further as Corollary 1) from Theorem 2. In [2] Theorem 2 for $n>2$ is reduced to this lemma, but the proof of the lemma is missing.

Corollary 1. If $A=\left(a_{i j}\right)_{i, j=1}^{m}$ is a matrix such that $a_{i j}>0$ for any $i, j \in I_{m}$, then there exists $\sigma \in S^{m}$ and $\lambda_{1}, \ldots, \lambda_{m}>0$ such that $\lambda_{j} a_{\sigma(j) j} \geq \lambda_{i} a_{\sigma(j) i}$ for any $i, j \in I_{m}$.

Proof. Without loss of generality it can be assumed that $\sum_{j \in I_{m}} a_{i j}=1$. Consider a simplex $S$ with facets $\left\{F_{i}\right\}_{i \in I_{m}}$ and the points $\left\{x_{i}\right\}_{i \in I_{m}} \subseteq S$ such that the row of $A$ is their barycentric coordinates.

By Theorem 2 there exists $\sigma \in S_{m}$ such that

$$
S \subseteq \bigcup_{i \in I_{m}} \operatorname{conv}\left(\left\{x_{i}\right\} \cup F_{\sigma(i)}\right) .
$$

We rearrange the points $\left\{x_{i}\right\}_{i \in I_{m}}$ to obtain $\left\{x_{i}^{\prime}\right\}_{i \in I_{m}}$, such that for any $i \in I_{m}$ we have $x_{i}^{\prime}=x_{\sigma^{-1}(i)}$.

Take the $\sigma^{-1}(i)$ th row of $A$ as the $i$ th row of $C$. Thus in $C$ the coordinates of $\left\{x_{i}^{\prime}\right\}_{i \in I_{m}}$ are in their respective rows and

$$
S \subseteq \bigcup_{i \in I_{m}} V_{i}, \quad V_{i}=\operatorname{conv}\left(\left\{x_{i}^{\prime}\right\} \cup F_{i}\right) .
$$

The sets $\left\{V_{i}\right\}_{i \in I_{m}}$ satisfy the conditions of Lemma 3. By this lemma there exists $y \in$ $\bigcap_{i \in I_{m}} V_{i}$ with barycentric coordinates $\left(y_{i}\right)_{i=1}^{m}$.

We rewrite the condition $y \in V_{i}, i \in I_{m}$, in the coordinate representation

$$
y_{i} c_{i j} \leq c_{i i} y_{j}, \quad \forall j \in I_{m} .
$$

Take the matrix $B=\left(b_{i j}\right)_{i, j=1}^{m}$ such that $b_{i j}=y_{i} c_{i j} / c_{i i}$. It is obtained from $C$ by multiplying rows by the numbers $y_{i} / c_{i i}(i=1, \ldots, m)$. Then we see that

$$
b_{i j}=\frac{y_{i} c_{i j}}{c_{i i}} \leq y_{j}=\frac{y_{j} c_{j j}}{c_{j j}}=b_{j j}, \quad \forall i, j \in I_{m} .
$$

Obviously, $B$ is the matrix we need.

We give another corollary of Theorem 2:

Corollary 2. Let $\left\{C_{i}\right\}_{i \in I_{m}}$ be a family of angles $\mathbb{R}^{2}$, let $O$ be their common vertex, and let each of them be less than $\pi$. Let $\left\{x_{i}\right\}_{i \in I_{m}} \subseteq \mathbb{R}^{2}$ and $\bigcup_{i} C_{i}=\mathbb{R}^{2}$. Then there exists $\sigma \in S^{m}$ such that

$$
\bigcup_{i \in I_{m}}\left(x_{i}+C_{\sigma(i)}\right)=\mathbb{R}^{2}
$$

Remark. In fact the angles may be allowed to equal $\pi$. 
Proof. We find a polygon $X$ with sides $\left\{s_{i}\right\}_{i \in I_{n}}$ such that $O \in$ int $X$ and there is an injection

$$
\iota: I_{n} \rightarrow I_{m}
$$

such that for any $i \in I_{n}$,

$$
s_{i} \subseteq C_{l(i)} .
$$

Apparently, $X$ can be found.

Now we apply Theorem 2 to the polygon $\lambda X(\lambda>0)$ and the set $\left\{x_{i}\right\}_{i \in I_{n}}$ : we will have a permutation $\sigma^{\prime}(\lambda) \in S^{n}$ such that

$$
X \subseteq \bigcup_{i \in I_{n}} \operatorname{conv}\left(x_{i} \cup \lambda s_{\sigma^{\prime}(\lambda)}\right) .
$$

Obviously, we may assume $\lambda_{k} \rightarrow \infty$ such that $\sigma^{\prime}$ is the same for any $\lambda_{k}$.

If $\lambda_{k} \rightarrow \infty$, we have in the limit

$$
\mathbb{R}^{2}=\bigcup_{i \in I_{n}}\left(x_{i}+C_{\iota\left(\sigma^{\prime}(i)\right)}\right) .
$$

We can prove the following by applying Theorem 3 :

Corollary 3. Let $\left\{C_{i}\right\}_{i \in I_{m}}$ be a family of angles in $\mathbb{R}^{2}$, let $O$ be their common vertex, and assume all angles to be less than $\pi$. Let $\left\{x_{i}\right\}_{i \in I_{m}} \subseteq \mathbb{R}^{2}$ and let int $C_{i}$ be mutually disjoint. Then there exists $\sigma \in S^{m}$ such that

$$
C_{i}^{\prime}=x_{i}+C_{\sigma(i)}
$$

have mutually disjoint interiors.

Proof. The proof is similar to that of Corollary 2; we have to find a polygon $X$ such that $O \in X$ and there is an injection

$$
\iota: I_{m} \rightarrow I_{n}
$$

such that for all $i \in I_{m}$,

$$
C_{i} \subseteq \operatorname{conv}\left(O \cup s_{l(i)}\right)
$$

It is natural to attempt to generalize these corollaries:

Question 1. Let $\left\{C_{i}\right\}_{i \in I_{m}}$ be a family of cones in $\mathbb{R}^{n}$ and let $O$ be their common vertex. Let $\left\{x_{i}\right\}_{i \in I_{m}} \subseteq \mathbb{R}^{n}$ and let $\bigcup_{i} C_{i}=\mathbb{R}^{n}$. Is it true that there exists $\sigma \in S^{m}$ such that

$$
\bigcup_{i \in I_{m}}\left(x_{i}+C_{\sigma(i)}\right)=\mathbb{R}^{n} ?
$$


Question 2. Let $\left\{C_{i}\right\}_{i \in I_{m}}$ be a family of cones in $\mathbb{R}^{n}$ and let $O$ be their common vertex. Let $\left\{x_{i}\right\}_{i \in I_{m}} \subseteq \mathbb{R}^{n}$ and let int $C_{i}$ be mutually disjoint. Is it true that there exists $\sigma \in S^{m}$ such that

$$
C_{i}^{\prime}=x_{i}+C_{\sigma(i)}
$$

have mutually disjoint interiors?

The following theorems can be proved similarly to Corollaries 2 and 3, they show that Questions 1 and 2 have positive answer under certain conditions:

Corollary 4. Question 1 can be answered positively if there is a polyhedral set $X$ with faces $\left\{F_{i}\right\}_{i \in I_{l}}$ and an injection

$$
\iota: I_{l} \rightarrow I_{m}
$$

such that for any $i \in I_{l}$,

$$
F_{i} \subseteq C_{l(i)},
$$

and $O \in \operatorname{int} X$.

Corollary 5. Question 2 can be answered positively if there is a polyhedral set X with faces $\left\{F_{i}\right\}_{i \in I_{l}}$ and an injection

$$
\iota: I_{m} \rightarrow I_{l}
$$

such that for any $i \in I_{m}$,

$$
C_{i} \subseteq \operatorname{conv}\left(O \cup F_{\iota(i)}\right)
$$

and $O \in \operatorname{int} X$.

Unfortunately, Questions 1 and 2 cannot be answered positively in the general case. This can be shown with a counterexample:

Take $\mathbb{R}^{3}$ with coordinates $\left(\xi_{1}, \xi_{2}, \xi_{3}\right)$. Denote

$$
\begin{aligned}
& C_{+}=\left\{\left(\xi_{1}, \xi_{2}, \xi_{3}\right): \xi_{1}, \xi_{2}, \xi_{3} \geq 0\right\}, \\
& C_{-}=\left\{\left(\xi_{1}, \xi_{2}, \xi_{3}\right): \xi_{1}, \xi_{2}, \xi_{3} \leq 0\right\}, \\
& C_{1}=\left\{\left(\xi_{1}, \xi_{2}, \xi_{3}\right): \xi_{1} \geq 0, \xi_{2} \leq 0\right\}, \\
& C_{2}=\left\{\left(\xi_{1}, \xi_{2}, \xi_{3}\right): \xi_{2} \geq 0, \xi_{3} \leq 0\right\}, \\
& C_{3}=\left\{\left(\xi_{1}, \xi_{2}, \xi_{3}\right): \xi_{3} \geq 0, \xi_{1} \leq 0\right\} .
\end{aligned}
$$

These five cones cover $\mathbb{R}^{3}$ and have mutually disjoint interiors. Consider some five points $\left\{x_{i}\right\}_{i \in I_{5}}$, all lying on the line $l$, defined by $\xi_{1}=\xi_{2}=\xi_{3}$. These five points and five cones give a negative answer to both Questions 1 and 2 . This can be seen if we notice that different lines, all parallel to $l$, intersect the cones in different orders. 
Now we state several conjectures:

Conjecture 1. Question 1 has a positive answer if the cones $C_{i}$ have mutually disjoint interiors and for every directed line l the cones can be ordered so that all lines, parallel to $l$, intersect the cones in the same order (dependent on the direction of $l$ ).

Conjecture 2. Question 2 has a positive answer if for every directed line l the cones can be ordered so that all lines, parallel to l, intersect the cones in the same order (dependent on the direction of $l$ ).

We formulate two other conjectures, which are particular cases of suppositions of Questions 1 and 2. They are interesting because they are formulated for the plane.

Conjecture 3. Let closed convex sets $\left\{A_{i}\right\}_{i \in I_{m}}$ partition the plane $\mathbb{R}^{2}$. Then for every set of vectors $\left\{v_{i}\right\}_{i \in I_{m}}$ there exists $\sigma \in S^{m}$ such that

$$
\mathbb{R}^{2}=\bigcup_{i \in I_{m}}\left(v_{\sigma(i)}+A_{i}\right)
$$

Conjecture 4. Let closed convex sets $\left\{A_{i}\right\}_{i \in I_{m}}$ partition the plane $\mathbb{R}^{2}$. Then for every set of vectors $\left\{v_{i}\right\}_{i \in I_{m}}$ there exists $\sigma \in S^{m}$ such that the sets

$$
A_{i}^{\prime}=v_{\sigma(i)}+A_{i}
$$

have mutually disjoint interiors.

Note that Corollaries 4 and 5 imply the above two conjectures if the sets $A_{i}$ are central projections of faces of a convex polytope.

If Conjectures 3 and 4 fail to be true, it is reasonable to ask about the necessary and sufficient conditions on $\left\{A_{i}\right\}$ for either of the conjectures to hold.

Corollary 4 helps us prove the following fact:

Corollary 6. Let $A=\left\{a_{i}\right\}_{i \in I_{m}}$ and $B=\left\{b_{i}\right\}_{i \in I_{m}}$ be subsets of $\mathbb{R}^{n}$, each containing $m$ points. Then there exists $\sigma \in S^{m}$ such that for any $i, j \in I_{m}$,

$$
\left(a_{i}-a_{j}, b_{\sigma(i)}-b_{\sigma(j)}\right) \leq 0 .
$$

Here $(\cdot, \cdot)$ is the scalar product. Inequality $\leq$ can be replaced with $\geq$ if we consider $-B$ instead of $B$.

Proof. First, we note that it is sufficient to consider the case $n=m-1$ : if $n<m-1$, then we can embed $\mathbb{R}^{n}$ into $\mathbb{R}^{m-1}$; if $n>m-1$, we can consider a projection $B^{\prime}$ of $B$ onto some $(m-1)$-dimensional affine subspace $L \supseteq A$ instead of $B$, since the scalar products are the same for $B^{\prime}$ as for $B$.

Now we should say that like in all other proofs of this paper we can consider $A$ to be in general position, that means that $A$ is a simplex in $\mathbb{R}^{m-1}$. Then consider the Voronoi 
regions for $A$ :

$$
C_{i}=\left\{x \in R^{m-1}: \forall j \neq i,\left|x-a_{i}\right| \leq\left|x-a_{j}\right|\right\}, \quad i, j \in I_{m} .
$$

In this particular case, $A$ is a simplex, hence the sets $C_{i}$ are simplicial cones, their common vertex being the center of the $(m-2)$-dimensional circumsphere of $A$. Take this center as the origin.

Note that the cones $C_{i}$ satisfy the conditions of Corollary 4. Indeed, take any $i \in I_{m}$. Let $n_{i}$ be the outer normal for the face of $A$ that does not contain $a_{i}$. Then it is easy to see that the ray starting from the origin in the direction of $n_{i}$ equals the intersection

$$
\bigcap_{j \neq i} C_{j} \text {. }
$$

Take some point $a_{i}^{\prime}$ on this ray and consider this done for all $i$. Then we can note that each face of the simplex $A^{\prime}=\operatorname{conv}\left\{a_{i}^{\prime}\right\}_{i \in I_{m}}$ is contained in the respective $C_{i}$ (each vertex of $A$, except $a_{i}^{\prime}$, is contained in $C_{i}$ ). That means that $C_{i}$ do satisfy the conditions of Corollary 4.

Now we apply Corollary 4 to the cones $C_{i}$ and the points $\left\{t b_{j}\right\}_{j \in I_{m}}$, where $t>0$ is a number to be determined later. We shall have $\sigma \in S^{m}$ such that the cones $C_{i}+t b_{\sigma(i)}$ cover $\mathbb{R}^{m-1}$.

Now consider the intersection of some pair: $C_{i}$ and $C_{j}$. This intersection $W_{i j}=C_{i} \cap C_{j}$ (a wall) is an $(m-2)$-dimensional convex polyhedral set, perpendicular to $\left[a_{i} a_{j}\right]$. Besides, the relative interior int $W_{i j}$ does not intersect any $C_{k}$, where $k \neq i, j$.

Take a point $p \in \operatorname{int} W_{i j}$. It has a neighborhood $U$ such that $\mathrm{cl} U$ does not intersect any $C_{k}$, except $C_{i}$ and $C_{j}$. It is clear that for small enough $t$ the sets $C_{k}+t b_{\sigma(k)}(k \neq i, j)$ do not intersect $U$ for any $\sigma$. For small enough $t, C_{i}+t b_{\sigma(i)}$ and $C_{j}+t b_{\sigma(j)} \operatorname{cover} U$ if and only if

$$
\left(t b_{\sigma(i)}, a_{i}-a_{j}\right) \leq\left(t b_{\sigma(j)}, a_{i}-a_{j}\right)
$$

(since $W_{i j} \perp a_{i}-a_{j}$ ), or else

$$
\left(a_{i}-a_{j}, b_{\sigma(i)}-b_{\sigma(j)}\right) \leq 0 .
$$

If we choose $t$ small enough for all pairs $i$ and $j$, the proof is complete.

S. E. Rukshin in a private talk with the author said that he has a proof of Corollary 1 by induction and a proof of Theorem 1, different from the above.

\section{Acknowledgment}

The author is very grateful to V. L. Dol'nikov for constant attention to this work and for useful discussions.

\section{References}

1. A. Bezdek, Covering a convex polygon by triangles (manuscript).

2. A. V. Bogomol'naya, F. L. Nazarov, and S. E. Rukshin, Covering a convex polygon by triangles with given vertices, Mat. Zametki 2 (1988), 191-195. 
3. A. Brønsted, An Introduction to Convex Polytopes, Springer-Verlag, New York, 1983.

4. L. Danzer, B. Grünbaum, and V. Klee, Helly's theorem and its relatives, in Convexity (V. Klee ed.), Proceedings of Symposia in Pure Mathematics, Vol. 7 American Mathematical Society, Providence, RI, 1963, pp. $101-181$.

5. R. Godement, Topologie algébrique et théorie des faisceaux, Hermann, Paris, 1958.

6. M. Hall, Combinatorial Theory, Blaisdell, Waltham, MA, 1967.

7. V. V. Proizvolov, Some unsolved problem of combinatorial geometry, Proceedings of First Soviet Conference on Combinatorial Geometry, Batumi, 1985, p. 54.

Received April 16, 2001, and in revised form October 11, 2001. Online publication March 1, 2002. 